\title{
Putting participants at the centre of HIV cure research
}

Curing HIV infection moved from the aspirational to the theoretically plausible with the apparent elimination of latent HIV from Timothy Ray Brown, also known as the Berlin Patient, after receipt of a stem cell transplant from a donor homozygous for the CCR5- $\triangle 32$ gene deletion. ${ }^{1}$ As HIV cure-related research has expanded, much of the science has centred on biomedical outcomes, and less on the people for whom a cure is being sought. Specifically, there has been limited inquiry directed towards understanding the motivations, perceptions, desires, needs, and experiences of people living with HIV who are asked to participate in cure-related research. This relative lack of attention is troubling when considering trade-offs, risks, demands, and burdens accompanying many of these investigations, such as intensively monitored antiretroviral pauses in effective HIV therapy, large volume blood draws and leucaphereses, and dosing with experimental immunotherapies.

The dearth of participant-centred data that come directly from people with HIV is particularly problematic given the expansion of the scope and scale of HIV curerelated research in recent years, ${ }^{2}$ which is being done against a background of highly effective, convenient, and well tolerated antiretroviral therapy (ART). Additionally, messaging regarding the importance of maintaining viral suppression, not only for personal wellbeing but also to practically eliminate the risk of transmission (ie, undetectable=untransmittable), can be undercut by designs calling for rebounds of viraemia. We need to understand participants' tolerance of viral
Published Online February 13, 2019 http://dx.doi.org/10.1016/ S2352-3018(19)30031-1 
rebounds from their own viewpoints and personal stresses of knowing they are no longer virally suppressed. At present, HIV cure-related investigations uniformly increase personal and public-health risks without directly benefiting study participants. ${ }^{3}$ However, for there to be sustained viral suppression of HIV infection, people with HIV will need to participate in clinical investigations that incrementally will achieve this aim. Understanding patient-participant preferences towards improvements to standard daily ART will become more crucial with the advent of long-acting injectable formulations.

As more HIV cure-related research is done, it will be crucial to determine how people with HIV perceive and understand these investigations-including risks and benefits of research and the fact that they will likely not be cured-in order to implement studies that are acceptable to people with HIV and reflect their priorities and concerns. This can be accomplished with the incorporation of social science research methods into clinical HIV cure investigations. For example, in clinical studies, participant-centred outcomes such as reports that are meaningful to those participating in research can be added to the typical health status and biomedical research surveys. Participant reports are useful in determining tolerance for risk over time, ${ }^{4}$ as well as acceptable risk-benefit profiles of new interventions, and can identify heterogeneity in motivations or preferences. ${ }^{5}$ Participant-centred outcomes supplement biological endpoints, facilitate detection of psychosocial, ethical, or practical issues, are widely accepted by HIV care providers, ${ }^{6}$ and can enhance shared decision making. Importantly, participantcentred approaches have had positive effects on participant-researcher interactions and can foster trust in the clinical research environment. ${ }^{8,9}$ Building trust can go a long way to encouraging under-represented groups, such as women and minorities, to participate in research. Participant-centred outcomes can also play an important part in understanding, in an era of well tolerated, single-tablet, potent ART, the extent to which people with HIV are willing to accept unfavourable riskbenefit ratios and trade-offs to advance biomedical HIV cure-related science.

Furthermore, by placing participants at the centre of the research, we can obtain a true appreciation for the extent that participants understand the research and its purpose. Few studies closely examine informed consent and lived experiences. ${ }^{10}$ As a cure for HIV is being increasingly considered within our grasp, understanding and managing expectations of those considering participation is essential, as is the meaningful involvement of people with HIV throughout the entire clinical research process. People living with HIV serve many important roles in clinical research, from participants, information providers, advisors, reviewers, and coinvestigators. ${ }^{11,12}$ HIV biomedical researchers and clinicians have much to gain from adopting communitybased participatory practices, engaging people with HIV as partners when designing and implementing HIV cure research protocols, particularly as the HIV cure field is moving towards opened analytical treatment interruptions requiring extended periods of viraemia. Involvement of people living with HIV in cure clinical studies must acknowledge them as experts and build long-term trust with the community.

HIV cure research offers long awaited hope for ending one of the worst global disease epidemics. As this work advances and reveals strategies for keeping latent HIV under control, we must not neglect the people in whom this virus resides. How people living with HIV look at these studies, decide to join them (or not), and are affected by participating, are essential elements needed to design future studies that are ethical, fully informed and acceptable to those we wish to cure.

*Karine Dubé, Liz Barr, David Palm, Brandon Brown, Jeff Taylor

University of North Carolina, Gillings School of Global Public Health, Public Health Leadership Program, Chapel Hill, NC, USA (KD); AIDS Clinical Trials Group Community Scientific Subcommittee Representative, Johns Hopkins University, Baltimore, MD, USA (LB); Global HIV Prevention and Treatment Clinical Trials Unit Community Advisory Board (DP), Collaboratory of AIDS Researchers for Eradication Community Advisory Board (DP, JT), University of North Carolina at Chapel Hill, Chapel Hill, NC, USA; Center for Healthy Communities, University of California, Riverside School of Medicine, Riverside, CA, USA (BB); HIV and Aging Research Project-Palm Springs, CA, USA (JT) karine_dube@med.unc.edu

We declare no competing interests. We are indebted to David Wohl (University of North Carolina, Chapel Hill) for insightful feedback on this manuscript. We are also grateful to Davey Smith and Sara Gianella from the University of California San Diego, whose work in the Last Gift study inspired this manuscript. We thank Jon Li (Harvard University), and Eileen Scully (Johns Hopkins University School of Medicine) for including sociobehavioral research and community representatives as coinvestigators as part of their AIDS Clinical Trials Group HIV cure-related research protocols.

1 Allers K, Hütter G, Hofmann J, et al. Evidence for the cure of HIV infection by CCR5 $\triangle 32 \Delta 32$ stem cell transplantation. Blood 2011; 117: 2791-99. 
2 Treament Action Group. Research toward a cure trials. http://www. treatmentactiongroup.org/cure/trials (accessed Jan 29, 2019).

3 Dubé K, Henderson GE, Margolis DM. Framing expectations in early HIV cure research. Trends Microbiol 2014; 22: 547-49.

4 Henderson GE, Peay HL, Kroon E, et al. Ethics of treatment interruption trials in HIV cure research: addressing the conundrum of risk/benefit assessment. J Med Ethics 2017; 44: 1-7.

5 Medical Device Innovation Consortium. Patient centered risk-benefit project report. Medical Device Innovation Consortium (MDIC). http://mdic. org/wp-content/uploads/2015/05/MDIC_PCBR_Framework_Proof5_Web. pdf (accessed Jan 29, 2019).

6 Simpson KN, Hanson KA, Harding G, et al. Patient Reported outcome instruments used in clinical trials of HIV-infected adults on NNRTI-based therapy: a 10-year review. Health Qual Life Outcomes 2013; 11: 1.

7 Velikova G, Booth L, Smith AB, et al. Measuring quality of life in routine oncology practice improves communication and patient well-being: a randomized controlled trial. J Clin Oncol 2004; 22: 714-24.
8 Schandelmaier S, Conen K, von Elm E, et al. Planning and reporting of quality-of-life outcomes in cancer trials. Ann Oncol 2015; 26: 1966-73.

9 Ong WL, Schouwenburg MG, van Bommel ACM, et al. A standard set of value-based patient-centered outcomes for breast cancer. JAMA Oncol 2016; 3: 1-9.

10 Kass NE, Chaisson L, Taylor HA, Lohse J. Length and complexity of US and international HIV consent forms from Federal HIV Network Trials. J Gen Intern Med 2006; 26: 1324-28.

11 Patient-Partner. Patient involvement in clinical research: a guide for patient organisations and patient representatives. https://www.geneticalliance.org. uk/media/1602/patientspartnerforpatientorgs.pdf (accessed Jan 29, 2019).

12 Shippee ND, Domecq Garces JP, Prutsky Lopez GJ, et al. Patient and service user engagement in research: a systematic review and synthesized framework. Heal Expect 2015; 18: 1151-66. 